The Astrophysical Journal, 201:352-365, 1975 October 15

(C) 1975. The American Astronomical Society. All rights reserved. Printed in U.S.A.

\title{
THE MOLECULAR CLOUD SAGITTARIUS B2
}

\author{
N. Z. SCOVILle \\ Owens Valley Radio Observatory, California Institute of Technology \\ P. M. SOLOMON \\ Earth and Space Sciences, State University of New York, Stony Brook \\ AND \\ A. A. Penzias \\ Bell Telephone Laboratories, Crawford Hill Laboratories, Holmdel, New Jersey \\ Received 1975 February 19; revised 1975 April 17
}

\begin{abstract}
The structure of the Sgr B2 molecular cloud has been studied by detailed and extensive mappings of the $\mathrm{CO},{ }^{13} \mathrm{CO}, \mathrm{CS}$, and $\mathrm{H}_{2} \mathrm{CO}(2-\mathrm{cm})$ transitions. The cloud consists of a dense core at the $\mathrm{OH}$ maser position plus a very large envelope 45 pc across. Sagittarius B2 is one of the most massive objects in the Galaxy, containing about $3 \times 10^{6} M_{\odot}$ - an estimate arrived at independently from calculated ${ }^{13} \mathrm{CO}$ column densities, from application of the virial theorem, and from far-infrared measurements. The molecular gas has an average temperature $T_{k}$ of $\sim 20 \mathrm{~K}$; that this is similar to the temperature of grains emitting far-infrared radiation is taken as evidence of near thermal equilibrium between dust grains and $\mathrm{H}_{2}$. The dynamics of the gas in the cloud are found to be dominated by large-scale systematic motions with velocity not a monotonic function of radius.

Subject headings: molecules, interstellar - nebulae
\end{abstract}

\section{INTRODUCTION}

The intense thermal radio source Sgr B2 is located at a distance of $10 \mathrm{kpc}$ in the nuclear region of our Galaxy. Accounting for its emission are at least seven compact $\mathrm{H}$ II regions, each intrinsically as bright as Orion A (M42), plus a diffuse component with $10^{3} M_{\odot}$ of $\mathrm{H}$ II. In this setting one finds what must be the most spectacular molecular cloud in the Galaxy.

Due to the large gas column densities nearly every molecular transition so far detected in interstellar clouds has been seen here-many have been seen only here. Excepting the maser emission of $\mathrm{OH}$ and $\mathrm{H}_{2} \mathrm{O}$, all the observed lines have exceptionally large velocity widths (up to $50 \mathrm{~km} \mathrm{~s}^{-1}$ for $\mathrm{CO}$ ) and most may be seen over a sizable region $\sim 6^{\prime}\left(1^{\prime}\right.$ subtends $2.9 \mathrm{pc}$ at $10 \mathrm{kpc}$ ) with velocity a strong function of position.

In an optimistic attempt to unravel the very complex physical and kinematic structure of this region we have mapped lines of $\mathrm{CO},{ }^{13} \mathrm{CO}, \mathrm{CS}$, and $\mathrm{H}_{2} \mathrm{CO}$ all at angular resolution $\leq 2^{\prime}$. The four transitions were selected each to reveal a different property of the molecular gas: the kinetic temperature from the $\mathrm{CO}$ line, the optical depth and gas density variations from ${ }^{13} \mathrm{CO}$ and $\mathrm{CS}$ lines, and the $\mathrm{H}_{2} \mathrm{CO}$ absorption line, to distinguish foreground velocities from those occurring behind the thermal source. The validity of such interpretations for each line is generally affirmed by the crude consistency of the final model with observations of far infrared and other molecular emission.

\section{OBSERVATIONS}

The $\mathrm{CO},{ }^{13} \mathrm{CO}$, and $\mathrm{CS}$ data were obtained in 1972 with the 36-foot antenna of the National Radio
Astronomy Observatory (NRAO) ${ }^{1}$ at Kitt Peak, Arizona. The beam efficiency $(\eta)$ and width (HPBW) were approximately 0.6 and $1^{\prime}$. Spectral resolution was provided by a 50 -channel filter bank with resolution 1.25 MHz; all spectra were then smoothed to a velocity resolution of $5 \mathrm{~km} \mathrm{~s}^{-1}$. Radial velocities are referred to the local standard of rest.

The receiver baseline was removed by observing an OFF position $5^{\circ}$ west of Sgr B2 and by frequency switching $60 \mathrm{MHz}$ toward higher radial velocity. The velocity at the center of the signal band was fixed at $75 \mathrm{~km} \mathrm{~s}^{-1}$, that in the reference about $160 \mathrm{~km} \mathrm{~s}^{-1}$ higher. From $\mathrm{CO}$ observations of the higher velocities (Scoville et al. 1974), we know that the strongest emission $\left(T_{A} \approx 1 \mathrm{~K}\right)$ in the reference band is at $180 \mathrm{~km} \mathrm{~s}^{-1}$. This emission, if present, will slightly distort the final $\mathrm{CO}$ profiles at $20 \mathrm{~km} \mathrm{~s}^{-1}$. Since we are mainly concerned with velocities above $50 \mathrm{~km} \mathrm{~s}^{-1}$, we have made no attempt to correct the spectra for reference band emission. No residual baselines were removed; the zero levels in the spectra are thought to be accurate to better than $0.5 \mathrm{~K}$.

The observations of the 2-cm $K$-doublet of $\mathrm{H}_{2} \mathrm{CO}$ seen in absorption against Sgr B2 were taken from a separate $\mathrm{H}_{2} \mathrm{CO}$ study. The equipment and methods of data reduction are described by Scoville and Wannier (1975). For the $\mathrm{H}_{2} \mathrm{CO}$ observations the $\mathrm{HPBW}$ was $2^{\prime}$ (with the 140-foot NRAO antenna) and the velocity resolution was reduced to $5 \mathrm{~km} \mathrm{~s}^{-1}$ in order to be comparable with the emission-line observations.

The observed grid centered on the $\mathrm{OH}$ maser

${ }^{1}$ Operated by Associated Universities, Inc., under contract with the National Science Foundation. 
position $\left(\alpha_{1950}=17^{\mathrm{h}} 44^{\mathrm{m}} 11^{\mathrm{s}}, \delta_{1950}=-28^{\circ} 22^{\prime} 30^{\prime \prime}\right), 1^{\mathrm{s}}$ east and $15^{\prime \prime}$ south of the 2 -cm continuum peak, was the same for all molecules. Spectra were taken along two perpendicular strips across Sgr B2 OH and over an $8^{\prime}$ by $8^{\prime}$ region with spacing $2^{\prime}$ in $\alpha$ and $\delta$. CS spectra were limited to a short north-south strip.

\section{ANALYSIS}

The equation of transfer for a plane parallel cloud of molecules which uniformly fills the telescope beam is

$$
\begin{aligned}
T_{A}{ }^{\prime} & =\frac{T_{A}}{\eta} \\
& =\left\{\frac{h v}{k\left[\exp \left(h v / k T_{\mathrm{ex}}\right)-1\right]}-T_{c}{ }^{\prime}-T_{\mathrm{BG}}{ }^{\prime}\right\}\left(1-e^{-\tau}\right),
\end{aligned}
$$

where $T_{A}$ is the antenna temperature, $\eta$ the beam efficiency of the antenna, and $T_{c}{ }^{\prime}$ and $T_{\mathrm{BG}}{ }^{\prime}$ are the intensities of the continuum source and the cosmic background expressed as equivalent Rayleigh-Jeans brightness temperatures. $T_{\text {ex }}$ and $\tau$ are the excitation temperature and optical depth of the molecular transition.

At $2 \mathrm{~cm}$ the continuum antenna temperature of Sgr B2 is still substantial $\left(T_{c}=3.6 \mathrm{~K}\right.$, see Fig. 1). Also, the excitation temperature of the 2-cm $\mathrm{H}_{2} \mathrm{CO}$ levels is low $\left(T_{\text {ex }}<2.74 \mathrm{~K}\right.$ in almost all clouds [Scoville and Wannier 1975]). The $\mathrm{H}_{2} \mathrm{CO}$ lines are therefore seen in absorption of both the cosmic background and the free-free radiation. To compensate for

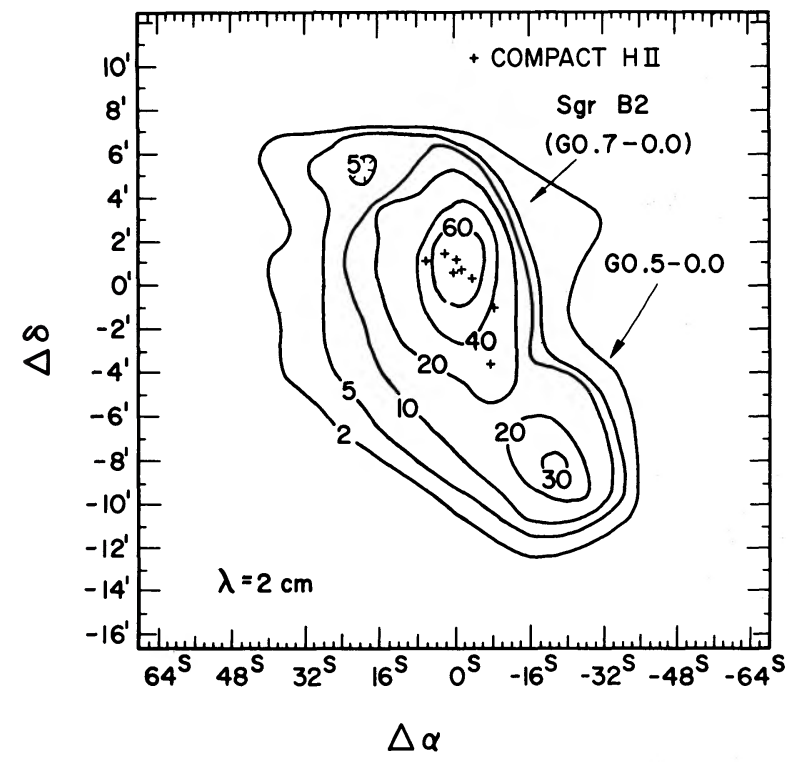

FIG. 1.-The brightness distribution of free-free radiation from Sgr B2 at 2-cm wavelength (HPBW $=2^{\prime}$; Scoville and Wannier 1975). Compact $\mathrm{H}$ iI regions detected by Martin and Downes (1972) and Hobbs et al. (1971) are shown. Coordinates are relative to the Sgr B2 OH maser $\left(\alpha_{1950}=17^{\mathrm{h}} 44^{\mathrm{m}} 11^{\mathrm{s}}\right.$, $\left.\delta=-28^{\circ} 22^{\prime} 30^{\prime \prime}\right)$, and the peak intensity (100 contour units) is $3.6 \mathrm{~K}, T_{A}$. the variation of $T_{c}$ across the region we convert the measured line intensities into optical depth measures using equation (1) and assuming that all the measured continuum originates from behind the cloud (Fig. 6). Since $T_{c}$ is much greater than $T_{\mathrm{BG}}$ over most of the Sgr B2 region, the more intense absorption will occur from molecules located in front of the $\mathrm{H}$ II regions; we use this strong bias to separate gas in front of the continuum source from that behind. Thus gas which appears strongly only in the emission lines and not in the $\mathrm{H}_{2} \mathrm{CO}$ absorption is very likely located behind the continuum sources.

Interpretation of the millimeter-wavelength emission lines is more intricate for, although the continuum intensity is negligible $\left(T_{c}=0 \mathrm{~K}\right)$, the excitation temperatures are strong functions of both velocity and position. In all sources where we have observed both the $\mathrm{CO}$ and ${ }^{13} \mathrm{CO}$ lines (or the $\mathrm{CS}$ and $\mathrm{C}^{34} \mathrm{~S}$ ) the rarer isotopes appear at a relative intensity much higher than their terrestrial abundance ratios. The relatively strong emission by the rare isotopes is an indication of high optical depths for the abundant species. ${ }^{2}$ The variation of the $\mathrm{CO}$ intensity within a given spectrum must then be due to velocity dependence of either $T_{\text {ex }}$ or the beam filling factor. Elimination of the latter possibility is virtually impossible, solely on an observational basis. A filling factor with Gaussian dependence on the velocity, as expected for randomly moving cloudlets, may easily be ruled out from the line shapes; but clearly this is an overidealized model. We adopt $T_{\text {ex }}$ velocity dependent as a working hypothesis, keeping in mind the reservation that the telescope beam may not be entirely filled at all velocities.

If the molecular excitation is caused only by collisions with $\mathrm{H}_{2}$, then all isotopes of a given molecule will have the same excitation temperature. The low intensity of a rare isotope (e.g., ${ }^{13} \mathrm{CO}$ ) as compared with the abundant species must then be taken as evidence of optical thinness (i.e., $\tau_{13 \mathrm{co}}<1$ ). However, if a substantial portion of the excitations in the abundant isotope is due to trapped line photons (see Scoville and Solomon 1974 and Goldreich and Kwan 1974), its excitation temperature will be greater than that of the rare isotope. One can then no longer calculate the optical depth of the rare isotope from the intensity ratio [e.g., $\left.T_{A}\left({ }^{13} \mathrm{CO}\right) / T_{A}(\mathrm{CO})\right]$. Trapping is likely to be especially important for molecules like CS whose large spontaneous rates $\left(A \sim 10^{-5} \mathrm{~s}^{-1}\right)$ would otherwise imply a high $\mathrm{H}_{2}$ density $\left(n_{\mathrm{H}_{2}}>10^{5} \mathrm{~cm}^{-3}\right)$ to produce the observed excitation. Our $\mathrm{C}^{18} \mathrm{O}$ observations in Sgr B2 suggest that radiative trapping may be important even for $\mathrm{CO}$ with its small dipole moment ( $\mu_{\mathrm{co}}=0.1$ debye) but high abundance.

In analysis of the $\mathrm{CO}$ and ${ }^{1 B} \mathrm{CO}$ data it is useful to assume that the $\mathrm{CO}$ species have identical excitation temperatures. The most abundant species ${ }^{12} \mathrm{C}^{16} \mathrm{O}$ is surely optically thick (see Penzias et al. 1971); the

${ }^{2}$ The alternative interpretation, that molecular isotopic abundances vary, seems contrived since the variation must occur within individual sources at different positions and velocities. 
Planck brightness temperature of $\mathrm{CO}$ (using eq. [5a] of Scoville and Solomon 1973) as a function of velocity and spatial coordinates is then equal to the $\mathrm{CO}$ excitation temperature. The optical depth of ${ }^{13} \mathrm{CO}$ lines in the unsaturated case can be calculated from their observed intensities as compared with the excitation temperatures which we take equal to the observed ${ }^{12} \mathrm{CO}$ brightness temperatures. We then calculate the molecular column density $N\left({ }^{13} \mathrm{CO}\right)$ in Figures 3 and 7 from

$$
N\left({ }^{13} \mathrm{CO}\right)=\frac{2.85 \times 10^{14} \tau \Delta v\left(T_{\mathrm{ex}}+1.0\right)}{1-\exp \left(-5.5 / T_{\mathrm{ex}}\right)} \mathrm{cm}^{-2},
$$

where $\Delta v[\mathrm{~km} / \mathrm{s}]$ is the full line width at half-intensity. ${ }^{3}$ When $T_{A}\left({ }^{13} \mathrm{CO}\right) \ll T_{A}(\mathrm{CO})$ and $T_{A}(\mathrm{CO})>5.5 \mathrm{~K}$, the calculated column density at any position and velocity will vary as the product of $T_{A}\left({ }^{13} \mathrm{CO}\right)$ and $T_{A}(\mathrm{CO})$.

The initial assumption that the two species $\mathrm{CO}$ and ${ }^{13} \mathrm{CO}$ have the same excitation temperature when combined with the relatively low ${ }^{13} \mathrm{CO}$ intensities implies that ${ }^{13} \mathrm{CO}$ is optically thin. A somewhat contrary situation is indicated by the dissimilarity of the ${ }^{13} \mathrm{CO}$ and $\mathrm{C}^{18} \mathrm{O}$ line shapes (Fig. 2) and by the narrow range of ${ }^{13} \mathrm{CO}$ optical depths from one source to the next implied if the ${ }^{13} \mathrm{CO}$ is optically thin. The central ${ }^{13} \mathrm{CO} / \mathrm{C}^{18} \mathrm{O}$ line intensity ratio is $\sim 5: 1$, i.e., about half the corresponding abundance ratio $\left[{ }^{13} \mathrm{C}\right][\mathrm{O}] /[\mathrm{C}]\left[{ }^{18} \mathrm{O}\right]$ (Gardner et al. 1971; Wannier et al. 1975), indicating a moderate $\tau=2$ saturation of the ${ }^{13} \mathrm{CO}$ line at its center. Though the ${ }^{13} \mathrm{CO}$ line is not optically thin over the entire area of the cloud, we can get an adequate approximation to the ${ }^{13} \mathrm{CO}$ column density by assuming that it is optically thin and strongly excited (i.e., its levels have an excitation temperature equal to that obtained for the corresponding CO line). The basis for this circumstance is that by using strong excitation and thus $\tau\left({ }^{13} \mathrm{CO}\right)<1$, one makes mutually canceling errors. As long as $\tau \leqslant 5$, the optical depth and ground-state population are underestimated by about the same factor that the partition function is overestimated. Consider a computation of the density at the Sgr B2 OH position (Fig. 2). At the velocity of $\mathrm{C}^{18} \mathrm{O}$ maximum, the $\mathrm{CO}$ and ${ }^{13} \mathrm{CO}$ temperatures $T_{A}{ }^{\prime}$ are $15 \mathrm{~K}$ and $8.7 \mathrm{~K}$, and the ratio $T_{A}{ }^{\prime}\left({ }^{13} \mathrm{CO}\right) / T_{A}{ }^{\prime}(\mathrm{CO})$ is 0.5 . If the ${ }^{13} \mathrm{CO}$ had the same excitation temperature as $\mathrm{CO}, \tau\left({ }^{13} \mathrm{CO}\right)=0.7$ and the computed column density for this optically thin ${ }^{13} \mathrm{CO}$ would be $2 \times 10^{16}$ $\mathrm{cm}^{-2} \mathrm{~km}^{-1} \mathrm{~s}$. If, instead, the ${ }^{13} \mathrm{CO}$ is optically thick, its excitation temperature is $8.7 \mathrm{~K}$. Application of equation (2) with the $\tau\left({ }^{13} \mathrm{CO}\right)=2$ as above yields once again the ${ }^{13} \mathrm{CO}$ column density of $2 \times 10^{16} \mathrm{~cm}^{-2}$ $\mathrm{km}^{-1} \mathrm{~s}$.

The above calculations serve only to illustrate that some optical depth in the ${ }^{13} \mathrm{CO}$ lines may be tolerated in using equation (2). When $\tau\left({ }^{13} \mathrm{CO}\right) \geqslant 10$, the errors introduced by an optically thin application of equation (2) will be large and grow in proportion to $\tau\left({ }^{13} \mathrm{CO}\right)$.

\footnotetext{
${ }^{3}$ Equation (2) differs from equation (7) of Scoville and Solomon (1973) in that a more precise evaluation of the partition function yields that +1.0 correction.
}

For high $\tau\left({ }^{13} \mathrm{CO}\right)$ but as long as the ${ }^{13} \mathrm{CO}$ intensities are subthermal [implied if $T_{A}\left({ }^{13} \mathrm{CO}\right)<T_{A}(\mathrm{CO})$ ], the variations of antenna temperature may still provide a crude deduction of gas density variations. The empirical results of molecular excitation calculations in this physical regime may be represented approximately by

$$
T_{\text {ex }} \propto\left(n^{13} \mathrm{Co}_{\mathrm{H}_{2}}\right)^{\sim 1 / 3} T_{K},
$$

a result applicable to clouds with $T_{K}>10 \mathrm{~K}$ and having large-scale motions (see Scoville and Solomon 1974). Assuming that the $\mathrm{CO}$ is thermalized and that the relative abundance ${ }^{13} \mathrm{CO}$ to $\mathrm{H}_{2}$ is constant, we find that maps of ${ }^{13} \mathrm{CO}$ and $\mathrm{CO}$ emissions may be converted to maps of molecular density as

$$
n_{\mathrm{H}_{2}} \propto n^{{ }^{13} \mathrm{CO}} \propto\left[T_{A}\left({ }^{13} \mathrm{CO}\right) / T_{A}(\mathrm{CO})\right]^{\sim 3 / 2} .
$$

Even if it is known that the radiative transfer model is appropriate, the utility of equation (4) is restricted to qualitative discussions because the exponent is variable between 1 and 2 . The implication is that the gas density distribution is a sharper function of both velocity and angular position that the observed ${ }^{13} \mathrm{CO}$ intensity distribution $\left[T_{A}(\mathrm{CO})\right.$ is usually slowly varying compared with $\left.T_{A}\left({ }^{13} \mathrm{CO}\right)\right]$.

The total hydrogen column density is estimated from $N\left({ }^{13} \mathrm{CO}\right)$ assuming an abundance ratio $\left[{ }^{13} \mathrm{C} / \mathrm{C}\right]=$ $1 / 50$ (Wannier et al. 1975) and $[\mathrm{CO} / \mathrm{H}]=\epsilon$; then

$$
N_{\Sigma \mathrm{H}}=\{50 / \epsilon\} N\left({ }^{13} \mathrm{CO}\right) \mathrm{cm}^{-2} \text {. }
$$

If all the carbon is bound up in $\mathrm{CO}$, then $\epsilon=3 \times 10^{-4}$ and $N_{\Sigma \mathrm{H}}=2 \times 10^{5} N\left({ }^{13} \mathrm{CO}\right)$; however, such high $\epsilon$ would imply very optically thick ${ }^{13} \mathrm{CO}$ lines in the observed clouds. More realistic estimates which are also consistent with equation (2) will be found using $N_{\mathrm{EH}}=2 \times 10^{6} N\left({ }^{13} \mathrm{CO}\right)$ corresponding to $\epsilon=3 \times 10^{-5}$ or 10 percent of all $\mathrm{C}$ in $\mathrm{CO}$.

\section{INTERPRETATION}

The observational results are displayed in two types of diagrams. The spatial-velocity maps depict changes in the line profile occurring as one scans across the cloud (Fig. 3 for $\mathrm{CO}$ and ${ }^{13} \mathrm{CO}$, and Fig. 4 for CS). The wealth of data, some of it irrelevant detail since the CO line is saturated, presented in this first type of diagram is often difficult to grasp. The spatial-spatial maps (Figs. 5, 6, and 7) depict the spatial dependence of a single parameter such as peak intensity. The second type of diagram is more comprehensible, but it also can give a deceptively simple impression of the gas distribution. Moreover, for such a complex cloud, some subjectivity is of course involved in choosing the parameter to be plotted, restricting the velocity range for measurement, and linking the contour segments.

In the following sections we discuss the temperature, gas density, and kinematics of the Sgr B2 cloudnecessarily avoiding much of the fine structure. Compact sources are clearly present (see Figs. 1 and 3 ) since Martin and Downes (1972) detect seven ionized regions with intrinsic properties equivalent to M42. However, 


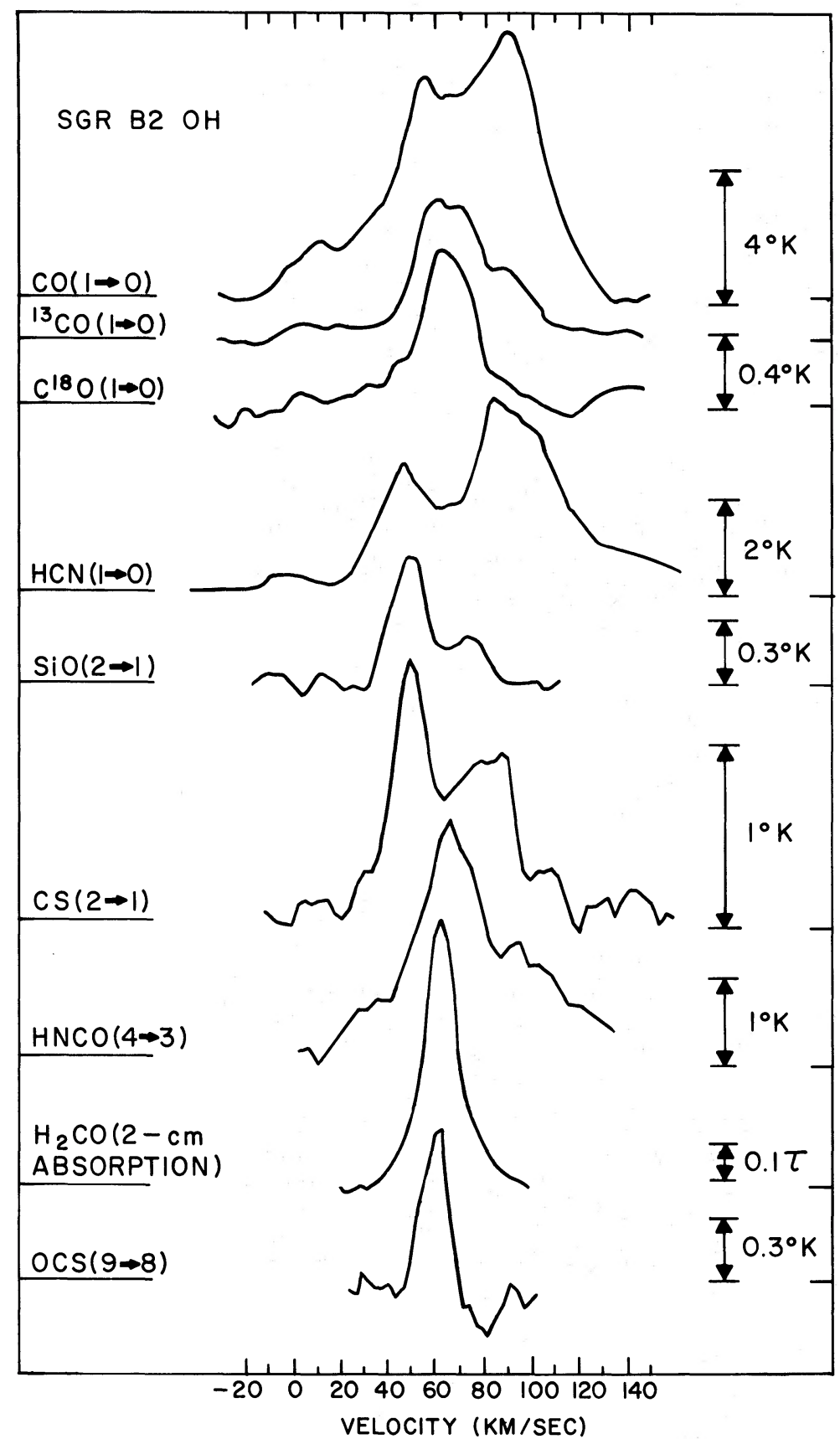

FIG. 2.-Molecular spectra in the direction of Sgr B2 OH. The antenna temperature scale is indicated to the right of each emission spectrum. Rayleigh-Jeans brightness temperatures $T_{A}^{\prime}$ are approximately $T_{A} / \eta_{B}=1.7 T_{A}$. The $2-\mathrm{cm} \mathrm{H}_{2} \mathrm{CO}$ line is seen in absorption of the Sgr B2 continuum radiation $\left(V_{\mathrm{H} 109 \alpha}=62 \mathrm{~km} \mathrm{~s}^{-1}\right.$, Fig. 1); its optical depth scale is indicated. All observations have an angular resolution of $1^{\prime}-2^{\prime}$ and velocity resolution $\leq 5 \mathrm{~km} \mathrm{~s}^{-1}$. Note the spike in the $\mathrm{C}^{18} \mathrm{O}$ spectra which is not seen in the more optically thick ${ }^{13} \mathrm{CO}$ and $\mathrm{CO}$ transitions. This narrow velocity distribution seems characteristic of optically thin molecular transitions in Sgr B2 (e.g., OCS and the 2-cm H $\mathrm{H}_{2} \mathrm{CO}$ line). The antithesis, the CS $2 \rightarrow 1$ line, not only is broad but may also show absorption by cold, foreground gas. (The temperature for the ${ }^{13} \mathrm{CO}$ spectrum is the same as that for $\mathrm{CO}$.) 

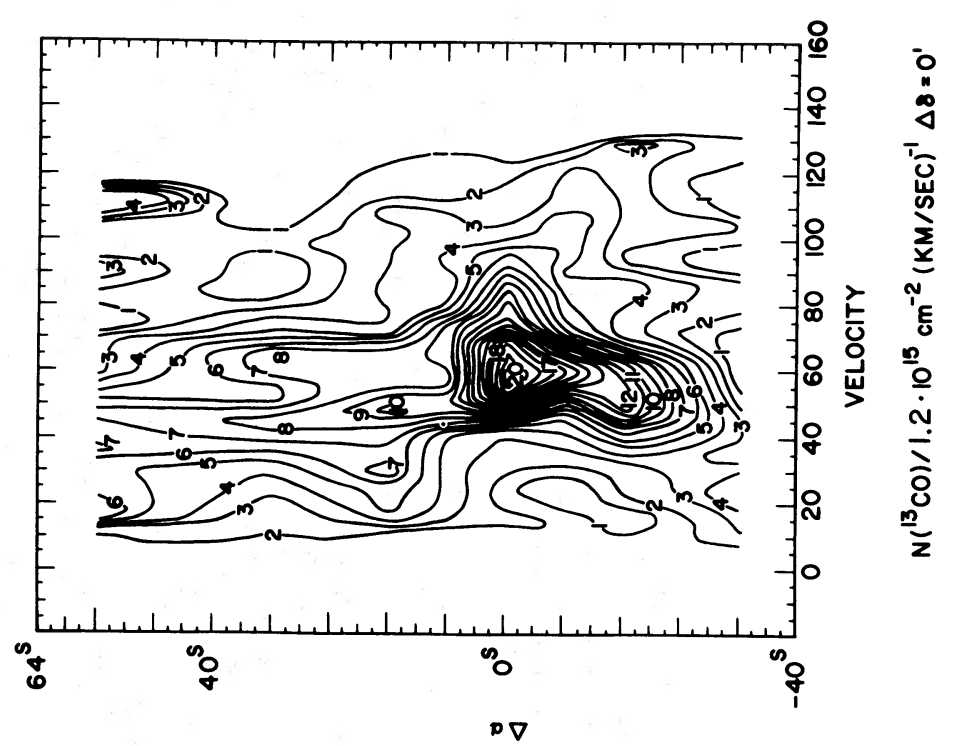

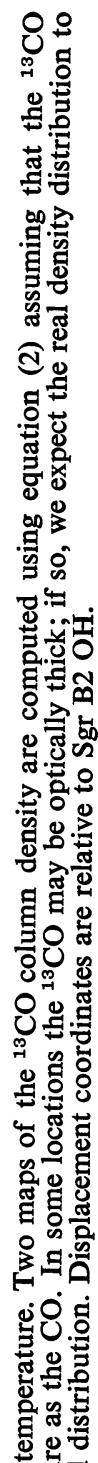

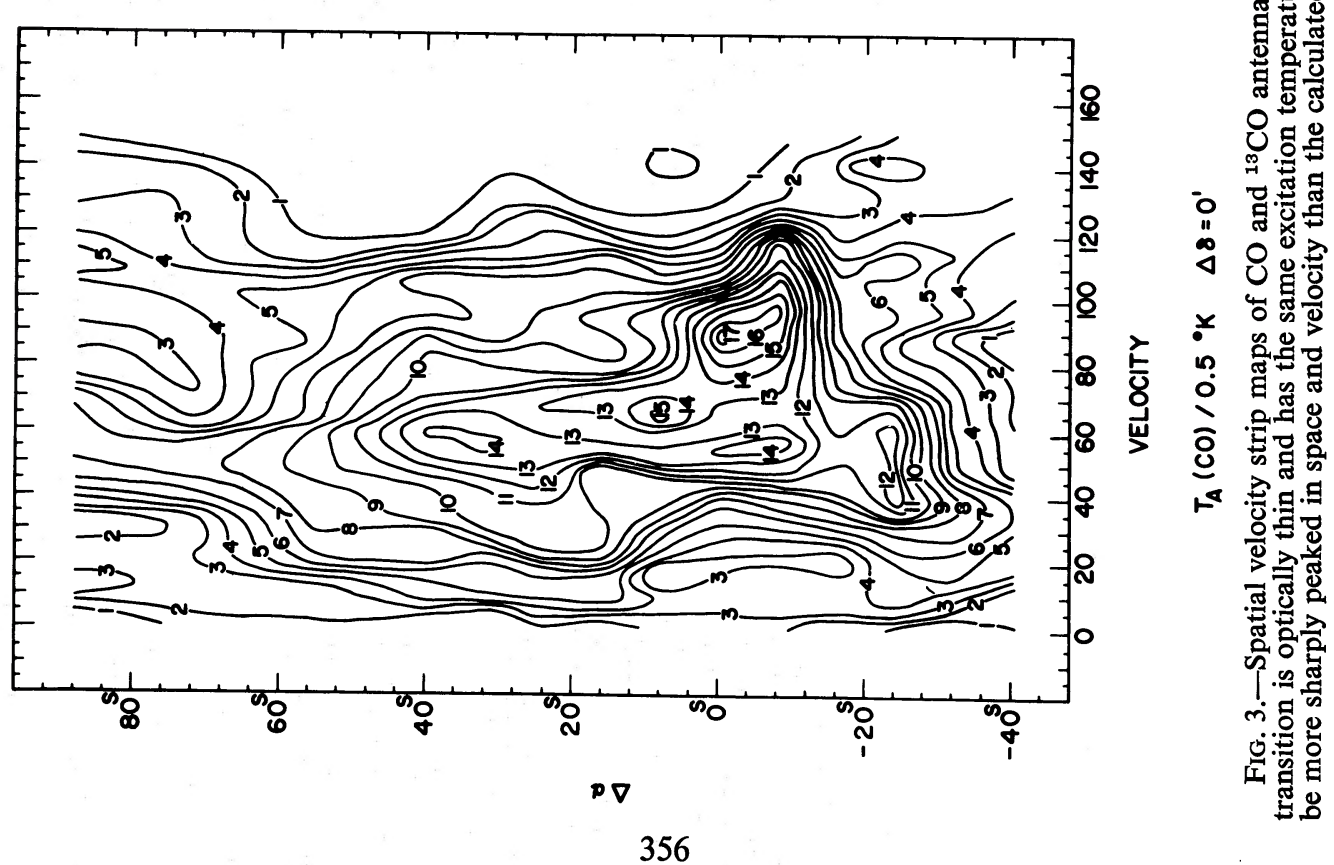



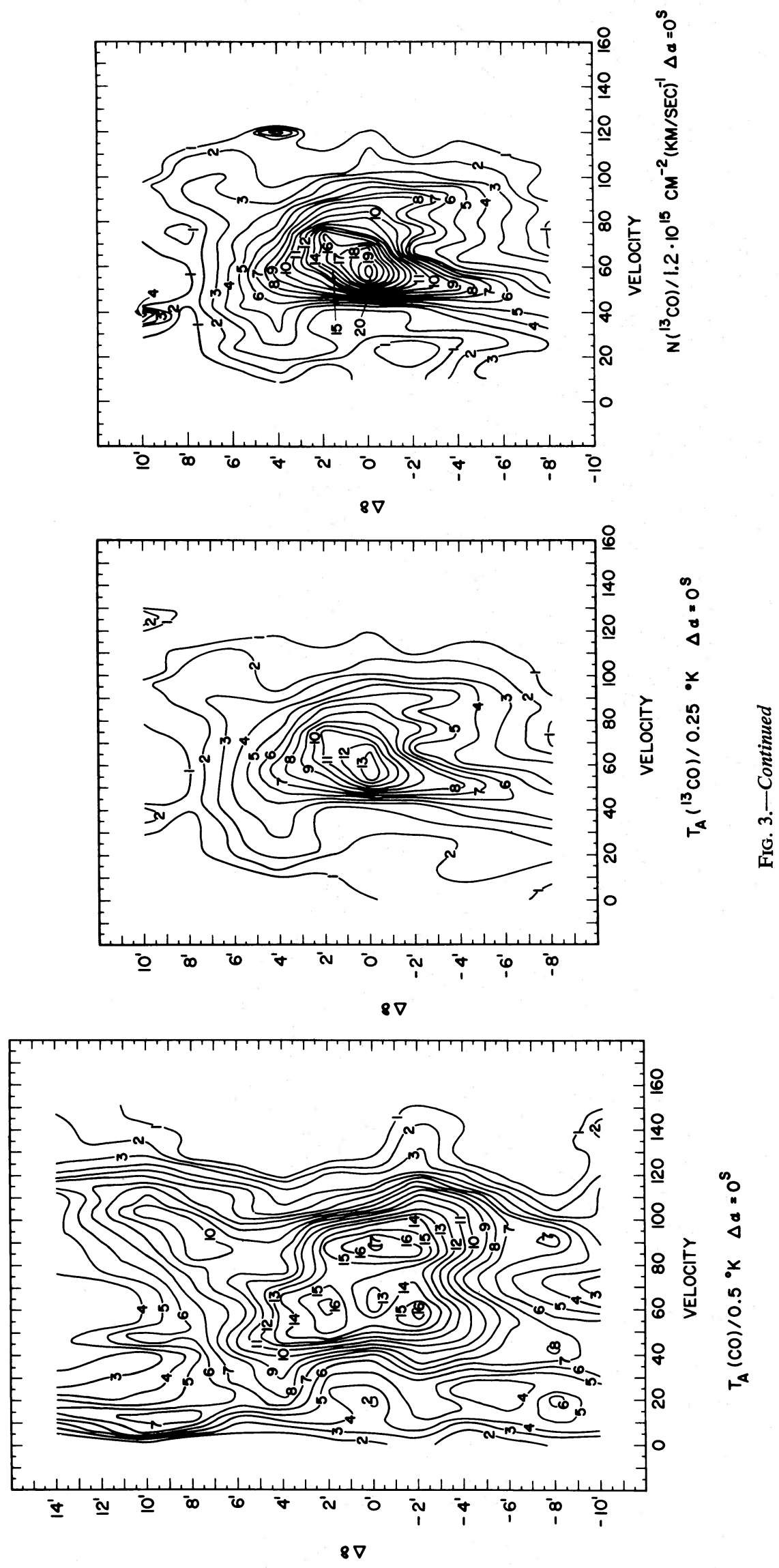


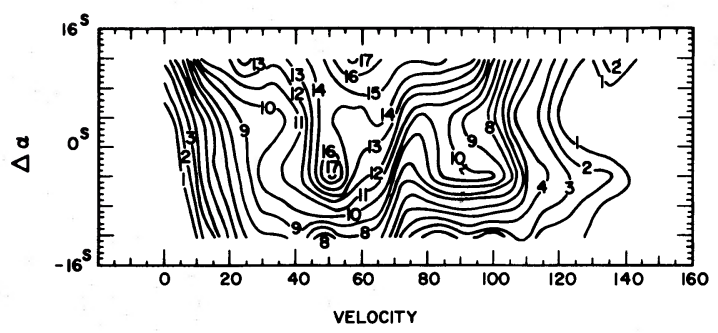

$T_{A}(C 0) / 0.5^{\circ} K \quad \Delta 8=+4^{\prime}$

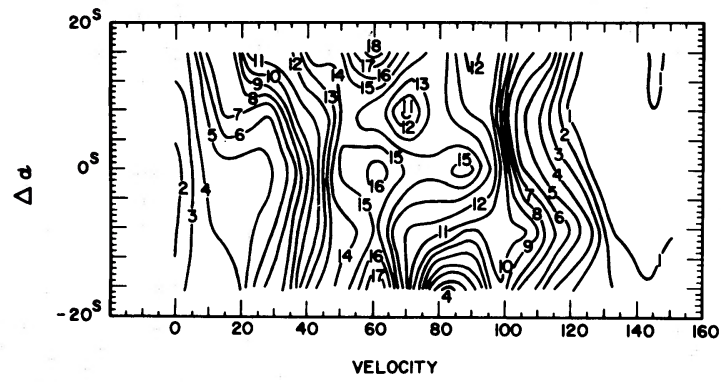

$T_{A}(\mathrm{CO}) / 0.5^{\circ} \mathrm{K} \quad \Delta 8=+2^{\prime}$

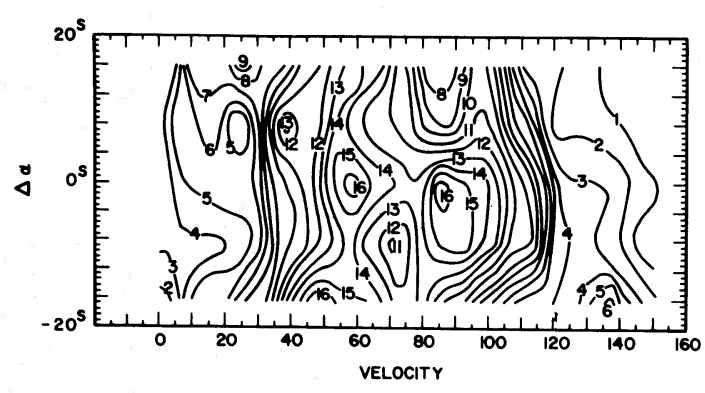

$T_{A}(\mathrm{CO}) / 0.5^{\circ} K \quad \Delta 8=-2^{\prime}$

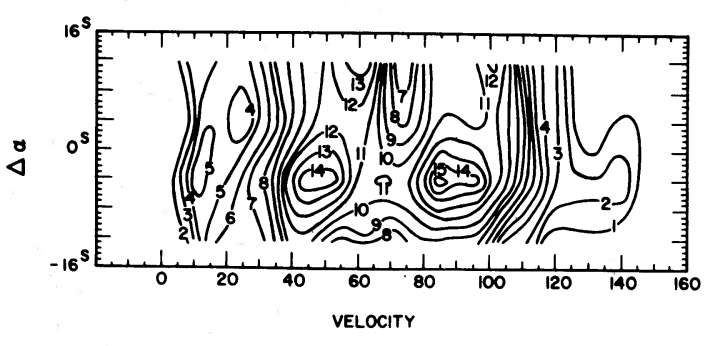

$T_{A}(c 0) / 0.5 \cdot k \quad \Delta 8=-4$

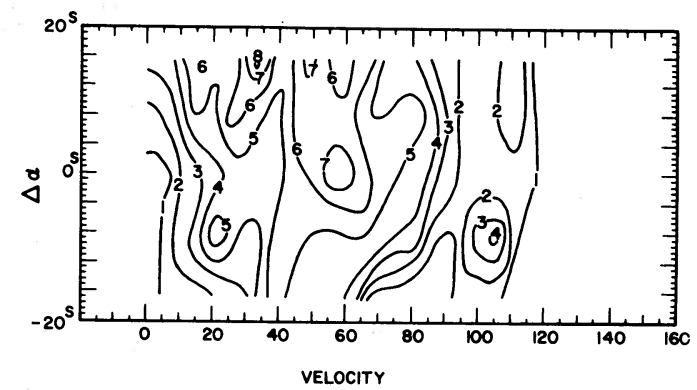

$T_{A}\left({ }^{13} \mathrm{Co}\right) / 0.25^{\circ} \mathrm{K} \quad \Delta 8=4^{\prime}$

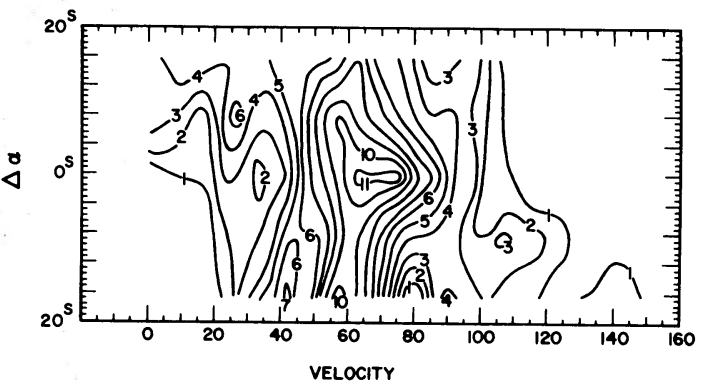

$T_{A}\left({ }^{13} \mathrm{CO}\right) / 0.25{ }^{\circ} K \quad \Delta \delta=+2^{\prime}$

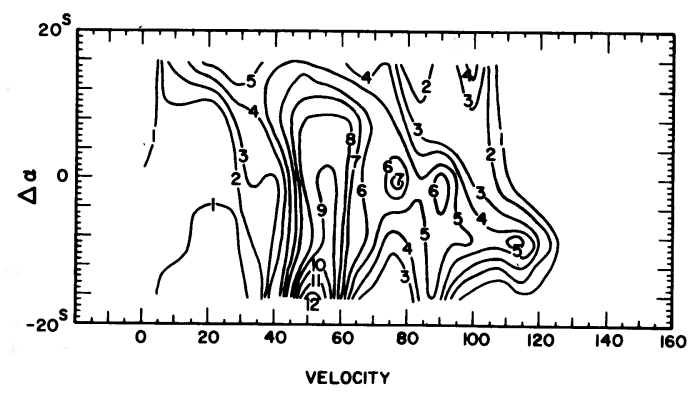

$T_{A}\left({ }^{13} \mathrm{Co}\right) / 0.25^{\circ} \mathrm{K} \quad \Delta 8=-2^{\prime}$

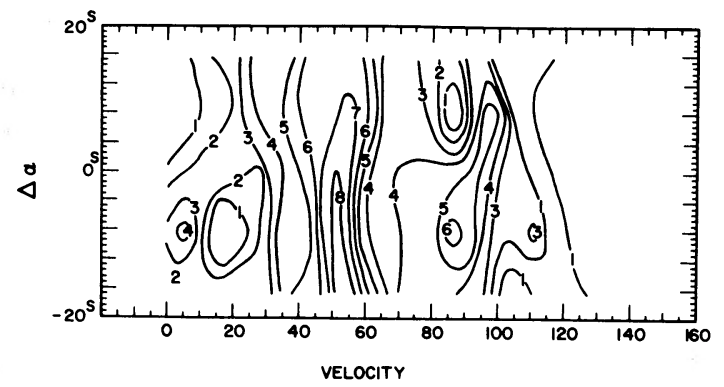

$T_{A}\left({ }^{13} \mathrm{Co}\right) / 0.250^{\circ} \mathrm{K} \quad \Delta 8-4^{\prime}$

Fig. 3.-Continued

a single source of high-intensity $\mathrm{CO}$ emission similar to the Kleinmann-Low nebula, removed to a distance of $10 \mathrm{kpc}$, would contribute negligibly to the observed emission due to beam dilution of its brightness $(\leqslant 2 \mathrm{~K})$ and small velocity width $\left(\sim 5 \mathrm{~km} \mathrm{~s}^{-1}\right)$.

\section{a) Velocity Structure}

The CO emission extends over a very wide velocity range (Fig. 2) with peaks at 53 and $90 \mathrm{~km} \mathrm{~s}^{-1}$ and a dip at $\sim 62 \mathrm{~km} \mathrm{~s}^{-1}$. In contrast, several other lines such as $\mathrm{OCS}, \mathrm{H}_{2} \mathrm{CO}$, or $\mathrm{C}^{18} \mathrm{O}$ show a single maximum at $v \approx 62 \mathrm{~km} \mathrm{~s}^{-1}$ and a much narrower width than either CO or ${ }^{13} \mathrm{CO}$. However, the HCN $(1-0)$, SiO $(2-1)$, and CS (2-1) show line profiles similar to CO with pronounced minima at this same velocity. The ${ }^{13} \mathrm{CO}$ and $\mathrm{HNCO}$ appear to have a width intermediate between these cases, with a single peak at $v \approx 62 \mathrm{~km}$ $\mathrm{s}^{-1}$ and an asymmetric shoulder extending out to 


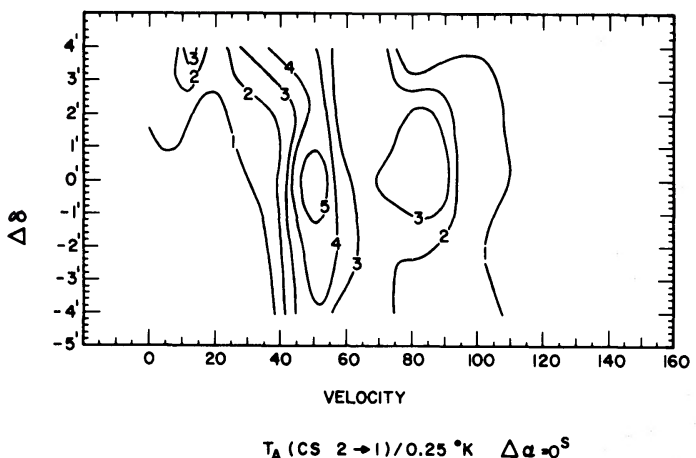

FIG. 4.-Declination strip map of the CS $J=2 \rightarrow 1$ antenna temperature.

$100 \mathrm{~km} \mathrm{~s}^{-1}$. The velocity spatial maps (Fig. 3) of the intensities show a complex double-peaked (in velocity) structure in $\mathrm{CO}$ and a single well-defined maximum at $60 \mathrm{~km} \mathrm{~s}^{-1}$ in ${ }^{13} \mathrm{CO}$. The abundance distribution of ${ }^{13} \mathrm{CO}$ in Figures 3 and 7 clearly shows a single welldefined feature.

The presence of a single peak in many of the weaker lines, particularly ${ }^{13} \mathrm{CO}$, suggests that the velocity distribution associated with Sgr B2 has but a single peak at approximately $62 \mathrm{~km} \mathrm{~s}^{-1}$ and a spatial distribution centered very near to the $\mathrm{OH}$ source. Thus, intensity peaks at 53 and $90 \mathrm{~km} \mathrm{~s}^{-1}$ could be due to absorption at $62 \mathrm{~km} \mathrm{~s}^{-1}$ from cool matter on the outside of the Sgr B2 molecule cloud with a similar velocity. From this point of view it is easy to understand why some molecules with high abundances $\mathrm{CO}, \mathrm{CS}, \mathrm{HCN}$, and $\mathrm{SiO}$ have minima at or near the velocity of the peak at $62 \mathrm{~km} \mathrm{~s}^{-1}$. These transitions have high optical depth in a medium with an excitation temperature gradient. The presence of this cool material in front of Sgr B2 at the same velocity as exists in the core is not surprising, since the core region presumably collapsed from a larger cloud. The fact that the 2-cm $\mathrm{H}_{2} \mathrm{CO}$ absorption line occurs at $65 \mathrm{~km} \mathrm{~s}^{-1}$ also suggests the presence of foreground matter at the velocity of the minimum in $\mathrm{CO}, \mathrm{CS}$, and $\mathrm{HCN}$.

The spatial variation of the peak intensities in the various transitions exhibit a dichotomy similar to the velocity distributions, giving further support to the contention that the brightness distributions of the higher optical depth transitions do not accurately reflect abundance variations. Thus the $\mathrm{CO}$ line is found to peak about $6^{\prime}$ northeast of the $\mathrm{OH}$ position while the ${ }^{13} \mathrm{CO}$ intensity peaks at the $\mathrm{OH}$ position and has a spatial distribution similar to the $\mathrm{H}_{2} \mathrm{CO}$ absorption (Fig. 5).

An exception to the suggested correlation between the presence of a dip in the Sgr B2 spectra and high optical depth transitions exists for the CS 1-0 transition. Besides the $J=2 \rightarrow 1 \mathrm{CS}$ transition (Fig. 2), the $J=1 \rightarrow 0$ (Turner et al. 1973) and $J=3 \rightarrow 2$ emissions have been observed in Sgr B2. Though the $J=3 \rightarrow 2$ transition shows the dip (Wilson et al. 1975), the $J=1 \rightarrow 0$ does not.

This effect may be explained by a density gradient with a corresponding decrease in collision rate in the outer part of the cloud. At low hydrogen density $n_{\mathrm{H}_{2}}<5 \times 10^{4} \mathrm{~cm}^{-3}$, with the $2.7 \mathrm{~K}$ background radiation field, the CS 2-1 line will be more optically thick, but at a lower excitation temperature than the 1-0 line (see Scoville and Solomon 1974). The optical depth ratio between two consecutive transitions $J+1 \rightarrow J$ and $J \rightarrow J-1$ is

$\frac{\tau_{J+1, J}}{\tau_{J, J-1}}=\frac{J+1}{J} \frac{1-\exp \left[-2(J+1) h B / K T_{J+1, J}\right]}{\exp \left(2 h B J / K T_{J, J-1}-1\right)}$,

which for CS 2-1, 1-0 at low $\mathrm{H}_{2}$ density gives $\tau_{2-1} /$ $\tau_{1-0}=1.2-3$. Thus absorption in the outer part of the Sgr B2 cloud will be more important for $J=2-1$ line than $J=1-0$. According to this explanation we expect that the rarer $\mathrm{C}^{34} \mathrm{~S}$ and ${ }^{13} \mathrm{CS}$ isotopes would show a single peak at the velocity of the dip in the $J=2 \rightarrow 1$ and $3 \rightarrow 2 \mathrm{CS}$ spectra. Indeed both these isotopes do show single peaks. In the $J=3 \rightarrow 2$ transitions the peak does occur about midway between the two peaks in the abundant isotope. The $J=2 \rightarrow 1 \mathrm{C}^{34} \mathrm{~S}$ peaks only $5 \mathrm{~km} \mathrm{~s}^{-1}$ higher than the low velocity CS peak rather than in the center of the dip as expected.

\section{b) Morphology}

The maximum $\mathrm{CO}$ and ${ }^{13} \mathrm{CO}$ intensities with their associated velocities are shown in Figure 3; the variations of the integrated intensities are similar. A truly striking aspect of the distributions, especially the $\mathrm{CO}$, is how smooth and extended they are. The distance, perpendicular to the galactic plane, between $\mathrm{CO}$ halfintensity points is $\sim 15^{\prime}$ amounting to $45 \mathrm{pc}$. Though more sharply peaked near the $\mathrm{OH}$ position, the ${ }^{13} \mathrm{CO}$ line is also seen over a similar area. The only other emissions mapped systematically here are the $3.4-\mathrm{cm}$ cyanoacetylene and the $1.3-\mathrm{cm} \mathrm{NH}_{3}$ transitions; in marked contrast to $\mathrm{CO}$, both have half-widths of only 4' (McGee et al. 1973; Cheung et al. 1969). However, since their excitation is poorly understood (and the $\mathrm{NH}_{3}$ observations are of low signal to noise), deduction of a reliable density distribution from the intensities is difficult.

There is an extended (over 30') background feature in the plane of the Galaxy from $l=0.4$ to $l=1: 1$ at $\sim 90 \mathrm{~km} \mathrm{~s}^{-1}$, which is apparent in maps of $\mathrm{CO}$ emission (Scoville et al. 1974) and $\mathrm{H}_{2} \mathrm{CO}$ and $\mathrm{OH}$ absorption (Scoville et al. 1972; Bieging 1974). Although this feature is also prominent near Sgr B2 (see Fig. 3), there does not appear to be any well defined spatial core associated with it. Emission is weak in the rarer isotopic species or less abundant molecules at this velocity. In particular, the absence of strong emission from ${ }^{13} \mathrm{CO}$ argues against this being a separate cloud associated with Sgr B2. We therefore ignore this $90 \mathrm{~km} \mathrm{~s}^{-1}$ feature in analyzing the Sgr B2 molecular cloud.

The variation of ${ }^{13} \mathrm{CO}$ column density computed using equation (2) and assuming identical $\mathrm{CO}$ and ${ }^{13} \mathrm{CO}$ excitation temperatures is shown in Figure 7. To avoid contamination by gas not associated with 


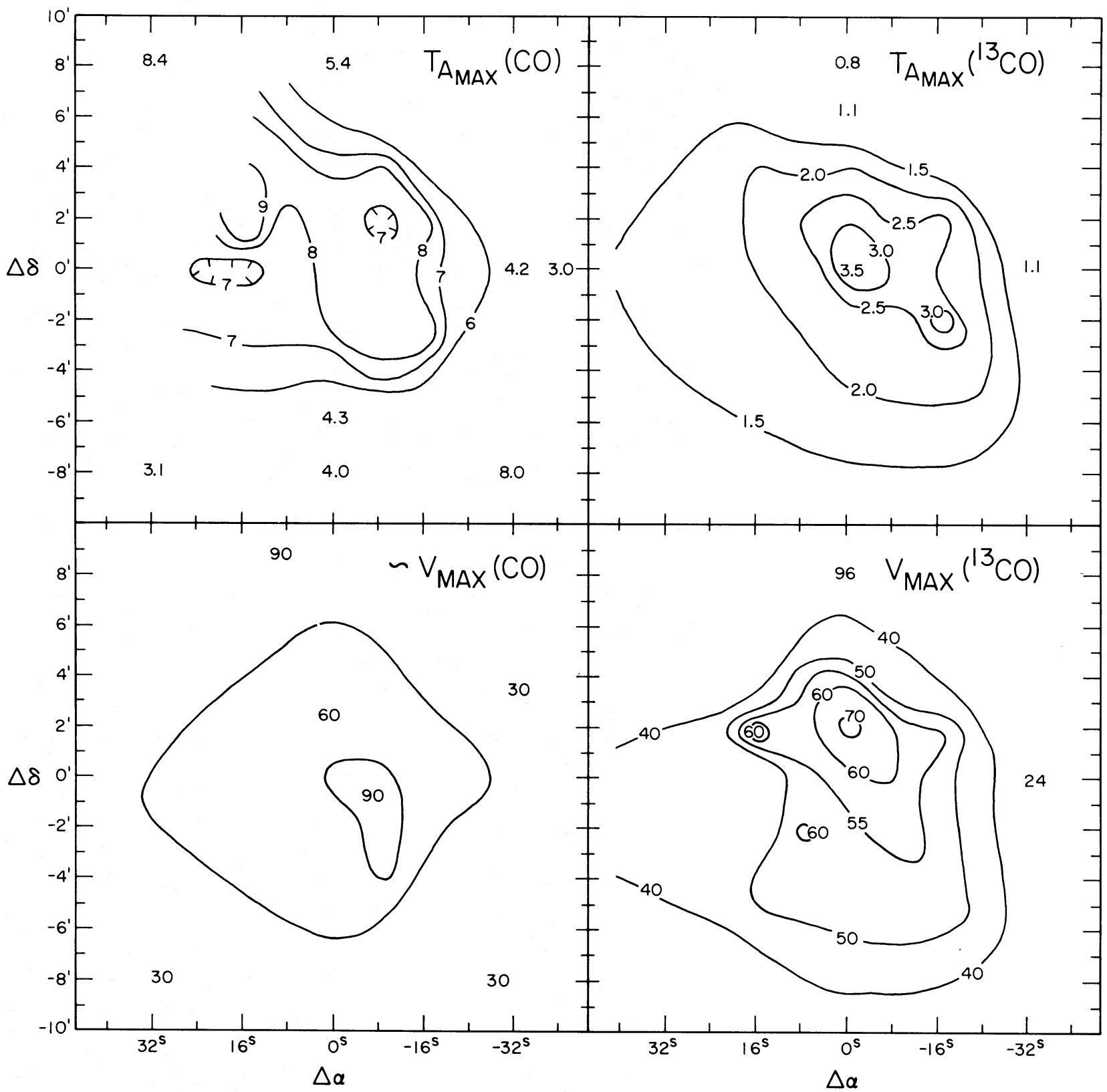

FIG. 5.-Approximate distributions of peak intensity and velocity of the $\mathrm{Sgr} \mathrm{B} 2 \mathrm{CO}$ and ${ }^{13} \mathrm{CO}$ emissions

Sgr B2, the velocity range of data used in this figure was restricted to $50-75 \mathrm{~km} \mathrm{~s}^{-1}$. This excludes contributions from the high-velocity $\mathrm{CO}$ feature in addition to low-velocity gas located outside the galactic center (these are at most 20 percent of the density between 50 and $75 \mathrm{~km} \mathrm{~s}^{-1}$; see Fig. 3). We feel that despite reservations about the assumption for the ${ }^{13} \mathrm{CO}$ excitation temperatures, these maps provide the most reliable indication yet for the mass distribution, free of bias toward high-excitation regions, free of bias toward gas situated on the near side of the continuum source, and relatively free of observational bias.

Both the maximum and integrated column densities in Figure 7 show a high-density core at the $\mathrm{OH}$ maser position. This is enveloped in a much larger cloud slightly elongated parallel to the galactic plane. The variation of $2-\mathrm{cm} \mathrm{H}_{2} \mathrm{CO}$ optical depth (Fig. 6) shows qualitative similarities to the $\mathrm{CO}$ distribution, although the detailed shape and gradients are quite different. There is good correspondence in the angular 


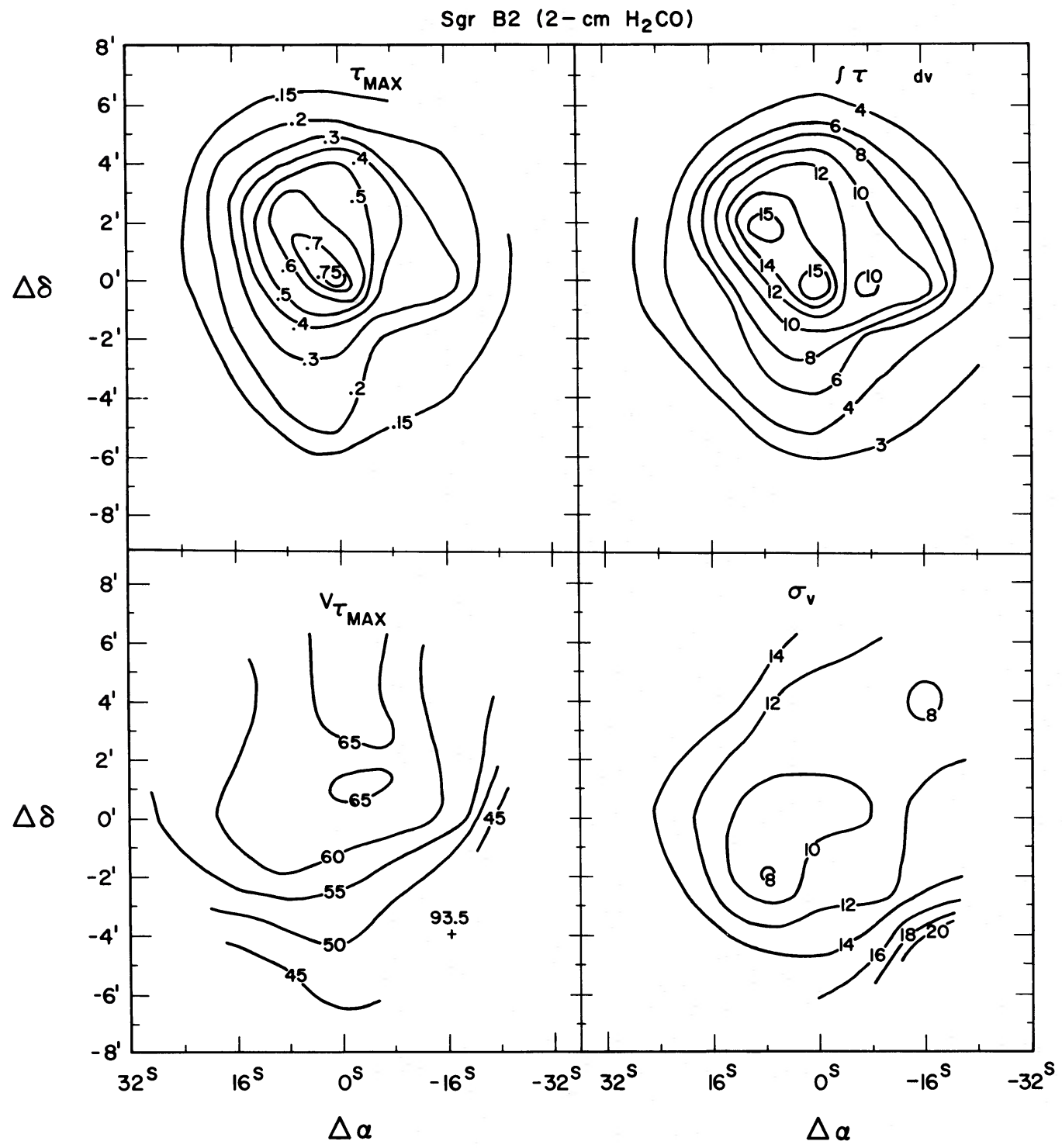

FIG. 6.-Contour diagrams of $\tau_{\max } \int \tau d v\left(\mathrm{~km} \mathrm{~s}^{-1}\right)$, velocity at $\tau_{\max }$, and velocity dispersion $\sigma_{v}$ for the 2-cm $\mathrm{H}_{2} \mathrm{CO}$ absorption between velocities 30 and $95 \mathrm{~km} \mathrm{~s}^{-1}$ (Scoville and Wannier 1975).

size and the peak locations-again at the $\mathrm{OH}$ position (see Fig. 6). The similarities between the $\mathrm{H}_{2} \mathrm{CO}$ absorption and the ${ }^{13} \mathrm{CO}$ maps suggests that most of the Sgr B2 molecular gas is situated on the near side of the diffuse $\mathrm{H}$ il region since any gas located on the far side would show strongly only in ${ }^{13} \mathrm{CO}$ emission. The low-intensity free-free radiation can be traced nearly as far out from the center as the $\mathrm{CO}$ emission, although its half intensity size $\left(3^{\prime}\right.$, Fig. 1$)$ is much less. All of the high-brightness, and presumably very young, $\mathrm{H}$ ir regions observed by Martin and Downes (1972) are clustered near the compact molecular component and the $\mathrm{OH}$ maser.

\section{c) Thermal Structure and the Far-Infrared Emission}

Due to the high abundance of $\mathrm{CO}$ the 1-0 transition is optically thick and nearly thermalized in this source. The $15-20 \mathrm{~K}$ brightness temperatures observed at the $\mathrm{OH}$ position are therefore indicative of the gas temperature averaged over the $1^{\prime}$ beam. Though a slightly higher temperature, $24 \mathrm{~K}$, is observed to the northeast, Figure 5 reveals little variation in the beamaveraged gas temperatures, aside from the gentle falloff at the edge of the cloud.

Goldreich and Kwan (1974) have considered the 


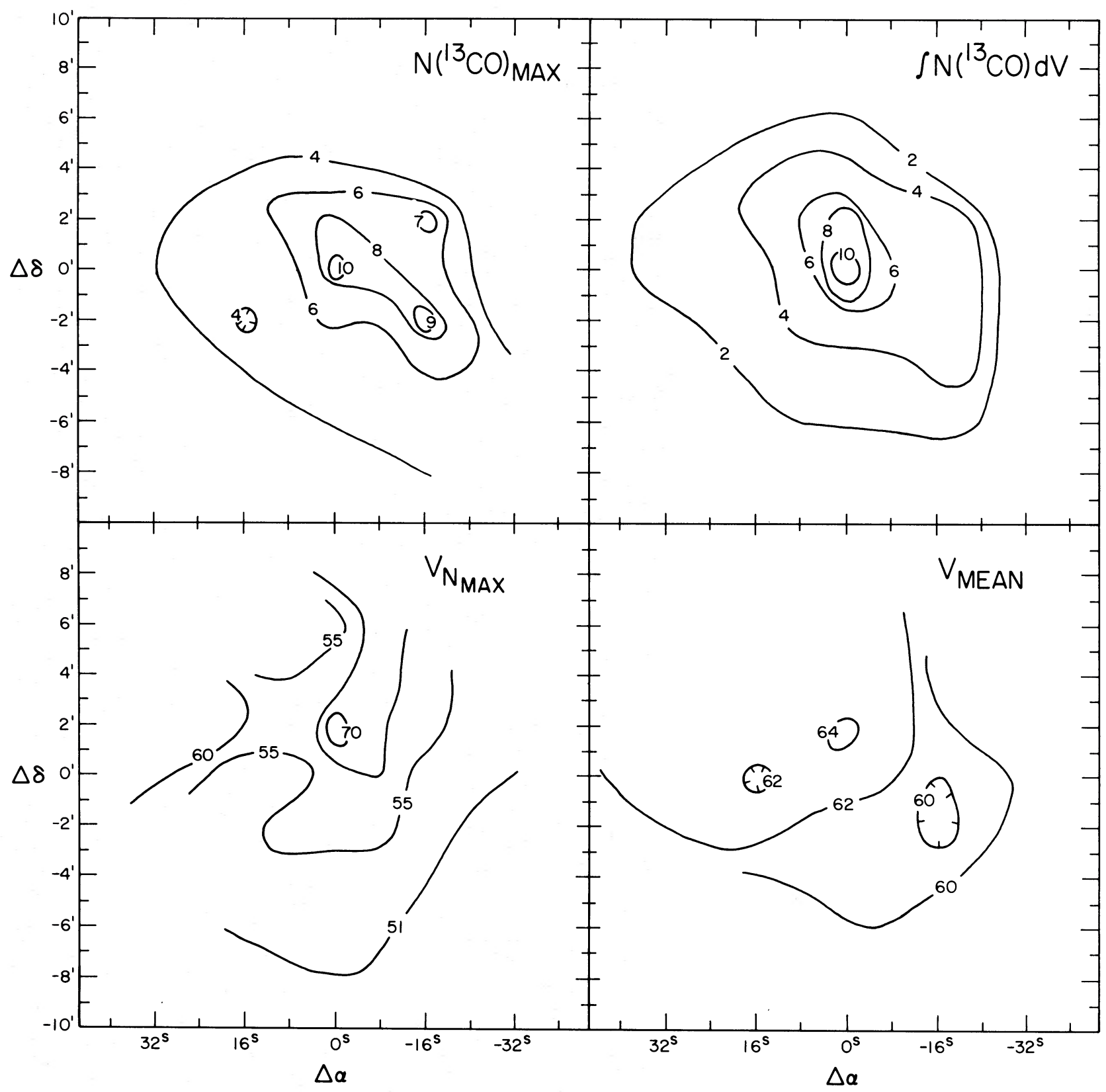

FIG. 7.-Contour diagrams of peak ${ }^{13} \mathrm{CO}$ column density (unit $=2.510^{15} \mathrm{~cm}^{-2} \mathrm{~km}^{-1} \mathrm{~s}$ ), the total ${ }^{13} \mathrm{CO}$ column density (unit $=$ $5.7 \times 10^{16} \mathrm{~cm}^{-2}$ ) between 50 and $75 \mathrm{~km} \mathrm{~s}^{-1}$, the peak and mean velocities. The best representation for the surface density of neutral gas and dust is probably given by $\int N\left({ }^{13} \mathrm{CO}\right) d v$. We find a compact, high-density component extending north about $2^{\prime}$ from Sgr B2 OH plus $a \sim 10^{\prime}$, low-density cloud.

thermal balance of molecular clouds similar to Sgr B2 when embedded luminous stars constitute the strongest heat source. Dust grains throughout the cloud are heated by the direct stellar radiation or by the secondary radiation of other heated dust if the cloud is optically thick at the frequency of maximum stellar radiation. The gas may then be thermally coupled to the warmer dust grains via collisions of $\mathbf{H}_{2}$ and grains
(Goldreich and Kwan 1974) or conceivably via radiation in far-infrared molecular transitions. The dust temperature $T_{D}$ is a very weak function of distance $R$ from the star. For a central energy source, it is well known that $\epsilon T_{D}{ }^{4} \propto R^{-2}$, where $\epsilon$ is the dust infrared emissivity. The wavelength dependence of the emissivity $\epsilon \propto \lambda^{-1} \rightarrow \lambda^{-2}$ can be combined with the Wien relation $\lambda_{\mathrm{MAX}} \propto T_{D}^{-1}$, to yield $T_{D} \propto R^{-2 / 5} \rightarrow R^{-2 / 6}$. 
If the collisional coupling of grains and gas is most important, the rate of gas heating will then be approximately given by

$$
\Gamma=\left[n n_{\text {grain }} \sigma_{\text {grain }} V\right] k \Delta T \approx 1.4 \times 10^{-33} n^{2} T^{1 / 2} \Delta T,
$$

where $\Delta T$ is the difference between grain and gas temperatures.

The cooling of the gas will be primarily by line radiation from abundant molecules. At low densities the cooling rate is of the form

$$
\Lambda \approx C_{1} n_{\mathrm{Co}} n T_{K}^{2} \text { for } n_{\mathrm{Co}} n_{\mathrm{H}_{2}} \ll 10^{2} \mathrm{~cm}^{-6} ;
$$

but at higher densities the dominant transitions thermalize and the cooling depends only on temperature as

$$
\Lambda \approx C_{2} T_{K}{ }^{3} \text { for } n_{\mathrm{co}} n_{\mathrm{H}_{2}} \gg 10^{2} \mathrm{~cm}^{-6}
$$

(Scoville and Solomon 1974). For cooling by $\mathrm{CO}$ and using a $\mathrm{CO}-\mathrm{H}_{2}$ total collisional cross section of $2 \times 10^{-15} \mathrm{~cm}^{2}, C_{1}=1.5 \times 10^{-28}$ and $C_{2}=2 \times 10^{-27}$ ergs $\mathrm{cm}^{-3} \mathrm{~s}^{-1}$. Equating the heating and cooling rates and restricting our attention to the high-density cooling law (7c), we find

$$
\Delta T \geq\left(10^{3} / n_{\mathrm{H}_{2}}\right)^{2} T_{K}^{5 / 2},
$$

where the inequality takes account of other molecular line coolants besides CO. From this relation we learn that the gas and dust temperatures will be equal to within 10 percent in the core where $n_{\mathrm{H}_{2}}=5 \times 10^{4}$ $\mathrm{cm}^{-3}$ (if $T_{D}=30 \mathrm{~K}$, then $T_{K}=28 \mathrm{~K}$ ) and in the envelope where $n_{\mathrm{H}_{2}} \approx 5 \times 10^{3} \mathrm{~cm}^{-3}$ (see $\S \mathrm{IV} e$ ), $T_{K}=12$ if $T_{D}=30 \mathrm{~K}$.

The near equality of the $\mathrm{CO}$ brightness temperature $(\sim 20 \mathrm{~K})$ and both the brightness and color temperatures of the far-infrared dust radiation $\left(T_{B}=15 \rightarrow 23 \mathrm{~K}\right.$, Righini et al. 1975 and $T_{\text {coLOR }}=33 \mathrm{~K}$, Harper 1974) suggests that the dust and gas are partially coupled.

In portions of the cloud where the dust is not heated much by radiation or where the atomic hydrogen fraction is much greater than 0.1 percent, an additional energy input may be provided by the heat of formation $(\sim 4 \mathrm{eV})$ of $\mathrm{H}_{2}$ on grains. If half of this exothermicity eventually goes into thermal energy of the gas (see Allen and Robinson 1975 for reservations concerning this assumption), it is easily shown that

$$
T_{\mathrm{K}}^{3 / 2} \approx 0.1 \frac{n_{\mathrm{H}}}{n} \frac{n}{n_{\mathrm{CO}}} \text { for } n_{\mathrm{Co}} n \ll 10^{2} \mathrm{~cm}^{-6}
$$

and

$$
T_{\mathrm{R}}{ }^{5 / 2} \approx 0.01 \frac{n_{\mathrm{H}}}{n} n^{2} \text { for } n_{\mathrm{CO}} n \gg 10^{2} \mathrm{~cm}^{-6},
$$

where $n \equiv n_{\mathrm{H}}+2 n_{\mathrm{H}_{2}}$. The ratio of gas heating by the hot dust and by $\mathrm{H}_{2}$ formation is approximately $5 \times 10^{-4} \Delta T n / n_{\mathrm{H}}$. This mechanism could therefore be important in the areas of Sgr B2 where the radiative heating of grains is small or in any cloud where significant atomic hydrogen still exists.
The spatial distribution for the far-infrared associated with the molecular cloud may be predicted from Figures 5 and 7. For wavelengths where the dust is optically thin and the Rayleigh-Jeans approximation is valid (i.e., $\lambda \geq 200 \mu$ for this cloud), the emission will vary as the product $T_{d} \tau$ which is presumably proportional to $T_{\mathrm{ex}}(\mathrm{CO}) \int N\left({ }^{13} \mathrm{CO}\right) d v$. Since the temperatures are nearly constant, we expect the farinfrared maps to look similar to the $\int N\left({ }^{13} \mathrm{CO}\right) d v$ plot (Fig. 7). At $350 \mu$ the strip scans of Rieke et al. (1973) show a peak near the $\mathrm{OH}$ position-extending $2^{\prime}$ north and 1' east-west. The more complete mapping of Righini et al. (1975) shows the same central peak plus an extended $\left(\sim 15^{\prime}\right)$, low-brightness (15 percent of peak) component which is possibly elongated parallel to the galactic plane. The good corresporidence between the far infrared maps and the ${ }^{13} \mathrm{CO}$ map in Figure 7 is evidence of the validity of the methods of analysis employed on both observations.

Within the compact component of the cloud, i.e., the density peak at the $\mathrm{OH}$ position, there may be temperatures much higher than $20 \mathrm{~K}$ in a region unresolved by the $1^{\prime}$ beam. Evidence for such higher temperatures is found in the emission observed by Solomon et al. (1971) from high levels of $\mathrm{CH}_{3} \mathrm{CN}$ which are metastable, populated only by collisions, and which therefore have relative populations characterized by $T_{K}$. At the $\mathrm{OH}$ position, and only there, emission with $T_{A} \approx 0.3 \mathrm{~K}$ is observed from levels at excitation energies equivalent to 77,127 , and $191 \mathrm{~K}$. Solomon et al. deduce that $T_{K} \approx 150 \mathrm{~K}$. However, this is akin to a "color" temperature; since the antenna temperature observed in a $1^{\prime}$ ' beam is much less $\left(T_{A}{ }^{\prime}=0.5 \mathrm{~K}\right)$, it is likely that the high temperature pertains to a small region. This explanation for the low brightness of the $\mathrm{CH}_{3} \mathrm{CN}$ emission nicely accounts also for the puzzling lack of any corresponding hightemperature $\mathrm{CO}$ emission. If the $\mathrm{CH}_{3} \mathrm{CN}$ lines are optically thick and the observed rotational transition $J=6 \rightarrow 5$ is thermalized, the approximate angular diameter of the hot core is $\sim 3^{\prime \prime}$. Unless this region were close to the near edge of the cloud, it would also not show up in infrared observations because of the overlying dust optically thick at $\lambda<100 \mu$.

\section{d) Dynamics}

On the large scale the center-of-mass motion of the cloud (relative to the galactic rest frame) is a combination of orbital and radial motions about the galactic center. The overall distribution of molecular gas in the nuclear region seems best fitted by a ring or spiral arm in which the Sgr B2 region is located 200 pc out from the galactic center (Scoville et al. 1974).

With regard to the internal motions of the cloud, all molecular lines in Sgr B2, and especially the CO lines, show an extraordinary range of macroscopic velocities (up to $50 \mathrm{~km} \mathrm{~s}^{-1}$ ). Whether these motions can be best described as random or structured is relevant to the evolutionary history of the cloud (e.g., collapse) and to a proper understanding of molecular excitation mechanisms. Lateral gradients in the radial velocity 
(across the face of the cloud) are comparable with the line width at any position (i.e., the range of velocities looking through the cloud. Moreover, at no point are the lines either Gaussian or even symmetric. These two simple observations constitute strong evidence that structured flows in the cloud are at least as large as random motions.

The actual percentage of the line width which can reasonably be attributed to random motions must be decided on the basis of plausibility arguments for the generation and maintenance of such "turbulence." For the Sgr B2 cloud it is possible to put firm upper limits on the permissible random motions. From the observed size and velocities we estimate the age of the region to be $\geqslant 10^{6}$ years. The maximum dissipation time for the turbulence will at most be the time needed to traverse the cloud $1 / f$ times while traveling at the turbulent velocity where $f$ is the fraction of the source area that is covered by the turbulent eddies (i.e., the filling factor). The high opacity of 6-cm $\mathrm{H}_{2} \mathrm{CO}$ and $18-\mathrm{cm} \mathrm{OH}$ absorption lines in front of Sgr B2 $\left(T_{A} / T_{c}>0.9\right.$ [Rogstad et al. 1974; Bieging 1974]) as well as the near equality of the $\mathrm{CO}$ brightness temperature and the far-infrared color temperature imply that $f \geqslant 1$ for this region. However, since the beam must be filled at each velocity within the line, the number of condensations with internal velocity dispersion $\Delta V_{\text {local }}$ along any line of sight through the cloud must be approximately $f\left\{\Delta V_{\text {line }} / \Delta V_{\text {local }}\right\}$. For thermal broadening of the individual elements we have $\Delta V_{\text {local }} \approx 0.3 \mathrm{~km} \mathrm{~s}^{-1}$. Thus, even for an $f$ as small as 0.5 we are led to conclude that the total number of condensations along any line of sight must be 100 .

The dissipation time for this turbulent model is then given by

$$
t_{d}=\left(D \Delta V_{\text {local }}\right) /\left(f \Delta V_{\text {line }}^{2}\right)
$$

where $D$ is the scale size, 3 pc for the core and 30 pc for the extended region. Substitution in this relation thus demonstrates the extremely short turbulent dissipation times, between $10^{3}$ and $10^{4}$ years. We therefore regard a predominantly turbulent model as inconsistent with the observations. A further discussion of the problem is presented elsewhere (Penzias 1975).

We are thus led to conclude that the line broadening is primarily due to structured motions. These flows are not very orderly, and there is little evidence that a coherent pattern is maintained across the entire cloud. In portions of the cloud steep lateral velocity gradients are seen (e.g., $\Delta \delta \approx 8^{\prime}$ in Fig. $3 a$ ) and a smaller $\mathrm{N}-\mathrm{S}$ gradient (Fig. 6) may be traced over the southern half of the cloud in most molecular lines. These gradients, combined with small density inhomogeneities, could easily be misinterpreted as multiple clouds in Sgr B2 if the molecular lines had not been mapped continuously over the region. Whether the gradients are due to rotational motion of the cloud or to radial motion which is not spherically symmetric is of course indeterminant from the present observations.

\section{e) Molecular Mass and Column Density}

Integrating over the ${ }^{13} \mathrm{CO}$ distribution (Fig. 7) and applying equation (5), we compute a total mass for the $50-75 \mathrm{~km} \mathrm{~s}^{-1}$ gas of $3 \times 10^{6} M_{\odot}$ with about 20 percent in the core. The hydrogen (nuclei) column density is $10^{24} \mathrm{~cm}^{-2}$, corresponding to a beam average volume density of $5 \times 10^{4} \mathrm{H}_{2} \mathrm{~cm}^{-3}$ within the core or $5 \times 10^{3}$ for the extended source. These values are based upon application of equation (2) to the data with the assumptions of optically thin ${ }^{13} \mathrm{CO}$, an abundance ratio $\left[\mathrm{C} /{ }^{13} \mathrm{C}\right]=50$, and 10 percent of the carbon in $\mathrm{CO}$.

Entirely independent estimation of the mass is arrived at by application of the virial theorem to the calculated velocity dispersions in $N\left({ }^{13} \mathrm{CO}\right)$ for which typically $\sigma=15 \mathrm{~km} \mathrm{~s}^{-1}$. We find that the mass required for stability of the cloud is $5 \times 10^{6} M_{\odot}$ if only the kinetic energy in the observed macroscopic velocities and gravitational energy are considered.

Finally we draw attention to the column densities deduced from the far-infrared opacity of the dust grains (Righini et al. 1975). If the grain temperature is given by the $50-300 \mu$ color temperature $(33 \mathrm{~K})$, then the optical depth of the cloud at $350 \mu$ is 0.4 . To relate this to the visual opacity we use the absorption curves measured by Knacke (1975) for silicate grains between $350 \mu$ and $2 \mu$ and then adopt a visual opacity of 10 times that at $2 \mu$ (Becklin 1974). This procedure yields a visual optical depth of $A_{V}=10^{3}$; taking a gas-to-dust ratio of $2 \times 10^{21} \mathrm{~cm}^{-2}$ per magnitude of visual extinction, the resulting $\mathrm{H}$ column density is $10^{24} \mathrm{~cm}^{-2}$. This is consistent with results of the molecular observations for $\epsilon=3 \times 10^{-5}$.

\section{CONCLUSIONS}

The Sgr B2 molecular cloud consists of a dense core $\sim 6$ pc running north from the $\mathrm{OH}$ source lying within an extended envelope of diameter 45 pc (at 20 percent the peak column density). The total mass of $\mathrm{H}_{2}$ here is estimated to be about $3 \times 10^{6} M_{\odot}$ on the basis of $\mathrm{CO}$ column densities, application of the virial theorem to the observed velocity dispersions, and far-infrared measurements. There is a single major peak at $62 \mathrm{~km}$ $\mathrm{s}^{-1}$ in the velocity distribution of molecules. The dip observed in line profiles of several high-optical-depth molecules at this velocity is probably not due to a minimum in the molecule column density and may be the result of absorption by cool foreground gas.

The gas temperature throughout most of the cloud is fairly constant at $\sim 20 \mathrm{~K}$. Since the brightness and color temperatures of the $350-\mu$ far-infrared radiation are similar to the above estimate which is based on $\mathrm{CO}$ observations, we suggest that the dust and gas are in near thermal equilibrium, coupled by collisions of $\mathrm{H}_{2}$ with dust grains. There is evidence of higher temperatures $\left(T_{K}>100 \mathrm{~K}\right)$ within a small region of the core source.

Large-scale motions dominate the velocity structure in Sgr B2. Strong gradients in the emission velocities are observed in scanning laterally across the face of the 
cloud (particularly in the north-south direction). These motions are, however, clearly more complex than a single expansion, contraction, or rotation of the cloud as a whole.

We are grateful to Peter Wannier for his aid in obtaining some of the spectra used in Figure 2 and to
John Kwan, Peter Goldreich, Mark Morris, and Dan Gezari for suggestions upon an earlier draft and to Claudine Gignoux for help in data reduction.

This research is sponsored in part by a National Science Foundation grant GP-30400-X5 and by the Office of Naval Research under contract N00 14-67-A0094-0019 at the Owens Valley Radio Observatory.

\section{REFERENCES}

Allen, C. W. 1963, Astrophysical Quantities (2d ed.; London: Athlone Press).

Allen, M., and Robinson, G. W. 1975, Ap. J., 195, 81.

Becklin, E. E. 1974, personal communication.

Bieging, J. 1974, unpublished Ph.D. thesis, California Institute of Technology.

Cheung, A. C., Rank, D. M., Townes, C. H., Knowles, S. H., and Sullivan, W. T. 1969, Ap. J. (Letters), 157, L13.

Gardner, F. F., Ribes, J. C., and Cooper, B. F. 1971, Ap. Letters, 9, 181.

Goldreich, P., and Kwan, J. 1974, Ap. J., 189, 441.

Harper, D. A. 1974, Ap. J., 192, 557.

Hobbs, R. W., Modali, S. B., and Maran, S. P. 1971, Ap. J. (Letters), 165, L87.

Knacke, R. $1975, A p . J$., in press.

Martin, A. H. M., and Downes, D. 1972, Ap. Letters, 11, 219.

McGee, R. X., Newton, L. M., Batchelor, R. A., and Kerr, A. R. 1973, Ap. Letters, 13, 25.

Penzias, A. A. 1975, Les Houches Lectures (North Holland, in press).

Penzias, A. A., Jefferts, K. B., and Wilson, R. W. 1971, Ap. J., 165, 229.

\section{A. A. Penzias: Crawford Hill HOH L239, Bell Telephone Laboratories, Holmdel, NJ 07733}

N. Z. Scoville: Department of Physics and Astronomy, University of Massachusetts, Amherst, MA 01002

P. M. Solomon: Earth and Space Sciences, State University of New York, Stony Brook, NY 11794
Rieke, G. H., Harper, D. A., Low, F. J., and Armstrong, K. R. 1973, Ap. J. (Letters), 183, L67.

Righini, G., Simon, M., Joyce, R., and Gezari, D. 1975, Ap. J. (Letters), 195, L77.

Rogstad, D. H., Lockhart, I. A:, and Whiteoak, J. B. 1974, Scoville, $\mathrm{N}$. Z., 36, 253.

Scoville N. Z., and Solomon, P. M. 1973, Ap. J., 180, 31. (Letters), 187, L63.

Scoville, N. Z., Solomon, P. M., and Thaddeus, P. 1972, Ap.J. $172,335$.

Scoville, N. Z., and Wannier, P. G. 1975, in preparation.

Solomon, P. M., Jefferts, K. B., Penzias, A. A., and Wilson, R. W. 1971, Ap. J. (Letters), 168, L107.

Turner, B. E., Zuckerman, B., Palmer, P., and Morris, M. 1973, Ap. J., 186, 123.

Wannier, P. G., Penzias, A. A., Linke, R. A., and Wilson, R. W. 1975, submitted to Ap. J.

ilson, R. W., Wannier, P. G., Linke, R. A., and Penzias, A. A. 1975 , in preparation. 